\title{
Magnetic and ferroelectric properties, crystal and magnetic structures of $\mathrm{SrFe}_{11.9} \mathrm{In}_{0.1} \mathrm{O}_{19}$
}

\author{
V.A. Turchenko ${ }^{1,2,3}$, A. Trukhanov ${ }^{4,5}$, S. Trukhanov ${ }^{2,4}$, F. Damay ${ }^{6}$, F. Porcher ${ }^{6}$, M. Balasoiu ${ }^{1,7}$, N. Lupu ${ }^{8}$, \\ H. Chiriac ${ }^{8}$, B. Bozzo ${ }^{9}$, I. Fina ${ }^{9}$, J. Waliszewski ${ }^{1,10}$, V.G. Kostishyn ${ }^{5}$, K. Recko ${ }^{10}$, S. Polosan ${ }^{11}$ \\ ${ }^{1}$ Department of Neutron Investigations of Condensed Matter, Joint Institute for Nuclear Research, Dubna, Russia; \\ ${ }^{2}$ Laboratory of crystal growth, South Ural State University, Chelyabinsk, Russia; \\ ${ }^{3}$ Department of Phase Transformation, Donetsk Institute of Physics and Technology named after O.O. Galkin of the NASU, Kiev, Ukraine; \\ ${ }^{4}$ Laboratory of magnetic films physics, SSPA Scientific and Practical Materials Research Centre of NAS of Belarus, Minsk, Belarus \\ ${ }^{5}$ Department of Electronic materials Technology, National University for Science and Technology MISiS, Moscow, Russia \\ ${ }^{6}$ Department of Powder diffractometers, Laboratoire Léon Brillouin, UMR12 CEA-CNRS, Bât. 563 CEA Saclay, France \\ ${ }^{7}$ Department of Nuclear Physics Department, Horia Hulubei National Institute for Physics and Nuclear Engineering, Bucharest, Romania \\ ${ }^{8}$ MDM Department, National Institute of Research and Development for Technical Physics, Iasi, Romania \\ ${ }^{9}$ Department of Magnetic Materials and Functional Oxides, Institut de Ciencia de Materials de Barcelona-CSIC, Barcelona, Spain \\ ${ }^{10}$ Faculty of Physics, University of Białystok, Białystok, Poland \\ ${ }^{11}$ Optical Processes in Nanostructured Materials Department, National Institute of Materials Physics, Bucharest-Magurele, Romania
}

E-mail: turchenko@,jinr.ru

Received xxxxxx

Accepted for publication $\mathrm{xxxxxx}$

Published xxxxxx

\begin{abstract}
The structural investigations of $\mathrm{SrFe}_{11.9} \mathrm{In}_{0.1} \mathrm{O}_{19}$ compound synthesized by solid-state method have been carried by neutron diffraction method in a wide temperature range. The appearace of spontaneous polarization that coexists with ferrimagnetic ordering has been found out at room temperature in strontium hexaferrite partially substituted with In ions. In order to explaine the presence of ferroelectric properties in $\mathrm{SrFe}_{11.9} \mathrm{In}_{0.1} \mathrm{O}_{19}$ compound, its crystal structure has been refined within the framework of both centrosymmetric $\mathrm{P}_{3} / \mathrm{mmc}$ (No. 194) and non-centrosymmetric $\mathrm{P}_{3} \mathrm{mc}$ (No. 186) space groups. The analysis of refinement results allows to understand microscopic mechanism of appearance ferroelectric properties in strontium hexaferrites.
\end{abstract}

Keywords: strontium hexaferrites, spontaneous polarisation, crystal and magnetic structures

\section{Introduction}

Solid solutions of M-type strontium ferrites belong to the extensive class of ferrimagnetic oxides with a rather complex crystalline structure. High values of the Curie temperature [1, 2] and saturation magnetization as well as high electrical resistivity and corrosion resistance allowed hexaferrites to receive a wide technical application as permanent magnets and magnetic information recording devices and microwave equipment. These materials are successfully used for electromagnetic shielding in the range of decimetric and centimetric radiowaves. Hexaferrite substrates with a high degree of magnetic texture are used to develop high-Q microband devices for microwave electronics.

It is well known that initial hexaferrites $\mathrm{MFe}_{12} \mathrm{O}_{19}(\mathrm{M}=$ $\mathrm{Ba}$ and $\mathrm{Sr}$ ) are isostructural to $\mathrm{PbO} \cdot 6 \mathrm{Fe}_{2} \mathrm{O}_{3}$ magnetoplumbite, which crystal structure was first 
determined by Adelskold [3] in 1938. There are two formula units $(Z=2)$ per unit cell of M-type hexagonal ferrite, i.e. the unit cell contains 2 strontium ions, 24 iron and 38 oxygen ions. The closest packing of $\mathrm{O}^{2-}$ ions forms several types of voids: octahedra $2 \mathrm{a}, 12 \mathrm{k}$ and $4 \mathrm{f}_{\mathrm{V}}$; tetrahedra $4 \mathrm{f}_{\mathrm{IV}}$ and trigonal bipyramid $2 b$, where magnetic Fe ions are located. Such a complex unit cell is characterized by significant crystalline anisotropy that is reflected in the ratio of the lattice parameters $\mathrm{c} / \mathrm{a} \approx 3.96$.

The magnetic structure of hexagonal ferrites is stipulated by superexchange interaction of iron ions through oxygen ions and forms a collinear ferrimagnetic ordering due to strong sublattice exchange.

As a rule, the crystal lattice is described within the framework of the centrosymmetric space group $\mathrm{P} 6_{3} / \mathrm{mmc}$ (No. 194). However, relatively recent studies of the interactions of barium and strontium hexaferrites with electric fields $[4,5]$ were discovered the presence of spontaneous electric polarization, its occurrence mechanism remains controversial. In the initial compounds of hexaferrites $\mathrm{MFe}_{12} \mathrm{O}_{19}(\mathrm{M}=\mathrm{Ba}$; $\mathrm{Sr}$ or $\mathrm{Pb})[4-6]$, the presence of nonzero spontaneous electric polarization has been explained by the distortion of a single oxygen octahedron $\mathrm{FeO}_{6}$ due to the displacement of the iron ion from the center [4, 7]. It was assumed that the distortion of a single oxygen octahedron $\mathrm{FeO}_{6}$ occurs below the Curie temperature. The appearance of ferroelectric properties in solid solutions of hexaferrites partially substituted with diamagnetic ions, such as $\mathrm{BaFe}_{12-\mathrm{x}-\delta} \mathrm{Sc}_{\mathrm{x}} \mathrm{Mg}_{\delta} \mathrm{O}_{19}(\mathrm{x}=1.6 ; \delta=$ 0.05 ) [8], is explained by the presence of a non-collinear magnetic structure. Moreover, a direct link between the electrical and magnetic subsystems was observed in Ref.[8]. As a result, taking into account a rather high temperature of the phase transition from ferrimagnetic to paramagnetic order, these compounds can be considered as promising multiferroics.

The present work continues the series our previous investigations [9-13], where we ascertain reasons of appearance spontaneous polarization in hexaferrites partially substituted with different diamagnetic ions (In, Ga, Al, Sc...). The main aim of this paper is to ascertain the microscopic mechanism of appearance of ferroelectric properties in the $\mathrm{SrFe}_{11.9} \mathrm{In}_{0.1} \mathrm{O}_{19}$ compound. The difference in the size of ionic radii In and Fe ions can be observed with diffraction methods that allows to evaluate influence of size effect to distortion internal structural elements. Moreover, the similar small concentration of In ions should not lead to appearance of non-collinear magnetic structure that allows to exclude its contribution to origin of spontaneous polarization. The present information is necessary to determine the formation mechanism of ferroelectric properties in hexaferrites.

\section{Measurement setup}

A polycrystalline sample $\mathrm{SrFe}_{11.9} \mathrm{In}_{0.1} \mathrm{O}_{19}$ was obtained by the solid-state reaction method. The initial oxides $\mathrm{Fe}_{2} \mathrm{O}_{3}$, $\mathrm{In}_{2} \mathrm{O}_{3}$ and carbonate $\mathrm{SrCO}_{3}$ were mixed in appropriate proportions and synthesized at $1200^{\circ} \mathrm{C}(6 \mathrm{~h})$ in air:

$$
\begin{gathered}
\mathrm{SrCO}_{3}+\left(6-\frac{1}{2} x\right) \mathrm{Fe}_{2} \mathrm{O}_{3}+ \\
\left(\frac{1}{2} x\right) \mathrm{In}_{2} \mathrm{O}_{3} \rightarrow \mathrm{SrFe}_{12-x} \operatorname{In}_{x} \mathrm{O}_{19}+\mathrm{CO}_{2} \uparrow
\end{gathered}
$$

The resulting composition was pressed and annealed at $1300^{\circ} \mathrm{C}(6 \mathrm{~h})$ and then slowly cooled $\left(\sim 100^{\circ} \mathrm{C} / \mathrm{h}\right)$. The Curie temperature of the sample was determined by the ponderomotive method by measuring its specific magnetization in the temperature range $300-800 \mathrm{~K}$ under external magnetic field of $0.86 \mathrm{~T}$.

Magnetization measurements were carried out on the MPMSXL superconducting quantum SQUID interferometer (DC magnetization mode) in the temperature range from 4 to $300 \mathrm{~K}$. Temperature dependences of magnetic susceptibility were measured by zero field cooled (ZFC) and field cooled warming (FCW) methods. In the first step, the sample was cooled without any magnetic field to $4 \mathrm{~K}$, while its heating was carried out with an applied magnetic field $\mathrm{H}=100 \mathrm{Oe}$. In the second step, the sample was cooled in an applied magnetic field of 100 Oe. In both cases, data were collected during heating the sample.

The geometry of a plane-parallel capacitor was given to the ceramic sample for measuring its polarization. The surface of strontium hexaferrite was coated with silver paste electrodes. Ferroelectric loops were measured using TFAnalyser 2000 (Aix-ACCT SystemsGmbH. Co.). The polarization was determined as the integral of current over time.

The absence of impurities in the sample was monitored by phase analysis on the X-ray diffractometer Empyrean (firm PANalytical) in Co- $\mathrm{K}_{\alpha}$-radiation.

The average crystallite size was determined by the broadening of diffraction peaks using the Scherrer's equation:

$$
<D>=\frac{K \lambda}{\beta \cos \theta}
$$

where $\mathrm{K}$ is a constant and characterizes the shape and size distribution of crystallites ( $\sim 0.94$ in the approximation of the spherical shape of crystallites), $\lambda$ is the $\mathrm{Co}-\mathrm{K} \alpha$ radiation wavelength, $\beta$ is the integral broadening of the diffraction peak at half its height, $\theta$ is the Bragg angle and $\angle D>$ is the average particle size in $\AA$.

Neutron diffraction studies of the sample in the temperature range from 1.5 to $350 \mathrm{~K}$ were carried out on the diffractometer G 4-1 in the experimental hall of the nuclear center in Laboratoire Léon Brillouin (LLB) (Saclay, France). 
The feature of the dual-axis neutron powder diffractometer $\mathrm{G}$ 4-1 is the 800 -channel detector $\left(\mathrm{BF}_{3}\right)$, which allows to cover a range of angles of $2 \Theta \sim 80^{\circ}$ and realize data acquisition at high speed. In addition, it realizes the ability to work in a monochromatic neutron beam with a wavelength of $2.426 \AA$. The measurements were carried out in a helium cryo refrigerator in the temperature range from 1.5 to $350 \mathrm{~K}$.

\section{Experimental results}

\subsection{Magnetic properties}

The temperature dependence of the specific magnetization of the $\mathrm{SrFe}_{11.9} \mathrm{In}_{0.1} \mathrm{O}_{19}$ ferrite is shown in Fig. 1. The Curie temperature of the compound is $\sim 670 \mathrm{~K}$, which is lower than the ferrimagnetic - paramagnet phase transition temperature $\mathrm{T}_{\mathrm{c}} \sim 732 \mathrm{~K}$ for the initial $\mathrm{SrFe}_{12} \mathrm{O}_{19}$ composition $[14,15]$. This behavior of the Curie temperature confirms the replacement of magnetoactive iron ions with diamagnetic indium ions. As a result, the magnetic order is destroyed at lower temperatures, which is consistent with the data [15, $16]$.

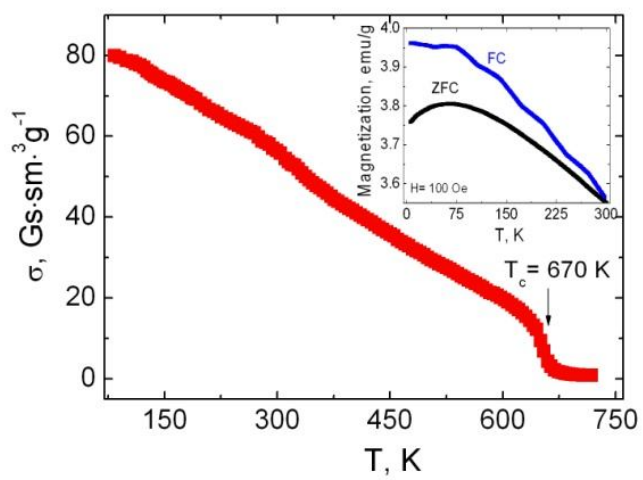

Figure 1. The temperature dependence of the specific magnetization of the $\mathrm{SrFe}_{11.9} \mathrm{In}_{0.1} \mathrm{O}_{19}$ compound. The Curie temperature is marked. The inset shows temperature dependences of the specific magnetizations of hexaferrite received in ZFC (blue line) and FCW (black line) mode.

The temperature dependencies of the magnetization of the $\mathrm{SrFe}_{11.9} \mathrm{In}_{0.1} \mathrm{O}_{19}$ compound collected in ZFC and FCW modes are shown in the insert Fig.1. The magnetization curve of ZFC is located below the FCW and first shows growth passing through the maximum, and then decreases when the sample is heated. The distinction between ZFC and FC curves was previously observed in ensembles of superparamagnetic particles [17], in spin glasses [18], as well as in macroscopic magnets with frustration of exchange bonds, with topological disorder, with structural defects, and even in ordered ferromagnets with significant magnetic anisotropy [19]. In our case, the sample can be represented as a system consisting of isolated particles with uniaxial anisotropy, in which the easy magnetization axes are randomly oriented in space. In a zero magnetic field, magnetizations of crystallites are ordered along their easy magnetization axes.
In the process of cooling the sample at $\mathrm{H}=0$, the magnetization of crystallites freezes at a certain blocking temperature $\left(T_{b}\right)$, while their distribution in the sample remains chaotic. The value of $T_{b}$ corresponds to the temperature below which the energy of thermal fluctuations in the crystallite is substantially less than the energy of the magnetic anisotropy. The value of blocking temperature can be determined by the Bean-Livingston equation (3) [20 - 22]:

$$
T_{B}=\frac{K_{V} V}{25 k_{B}}
$$

The presence of a broad maximum on the ZFC curve (see inset Fig.1) indicates the dispersion of particle sizes and it can be the result of the sum of narrow maxima for particles with various sizes. Therefore, the maximum is determined by the contribution of the volume fractions of particles with a certain size. When the sample is cooled in the magnetic field, the direction of the magnetization in each crystallite is determined by the competition between the magneto crystalline anisotropy energy and Zeeman energy. The magnetization of crystallites tends to be oriented in the direction of the external magnetic field. However, in order to rotate the magnetic moment of a particle in the direction corresponding to the minimum of energy, it is necessary to overcome the energy barrier:

$$
\Delta E \approx K_{V} \times V
$$

where $\mathrm{K}_{\mathrm{V}}$ is the volume anisotropy constant; $\mathrm{V}$ is the volume of the magnetic particle, which should be overcome by magnetic field in order to change the orientation of the magnetic moment of the particles.

At a sufficiently large intensity of the external magnetic field, magnetic moments of all crystallites are aligned along the field, and the shape of the temperature dependence curve in this case is determined by an increase in the spontaneous magnetization of the material.

The magnetic hysteresis loops of the $\mathrm{SrFe}_{11.9} \mathrm{In}_{0.1} \mathrm{O}_{19}$ compound measured at $\mathrm{T}=5 \mathrm{~K}$ and $300 \mathrm{~K}$ are shown in Fig. 2. The magnitude of the residual magnetization $M_{r}$, measured after switching off the external magnetic field, reaches values of $85 \mathrm{emu} / \mathrm{g}$ and $74 \mathrm{emu} / \mathrm{g}$ at temperatures $5 \mathrm{~K}$ and $300 \mathrm{~K}$, respectively. A similar difference in the residual magnetization values is the result of a decrease in the spin collinearity degree of $\mathrm{Fe}^{3+}$ at $300 \mathrm{~K}$ in particles due to an increase in temperature fluctuations [23, 24]. 


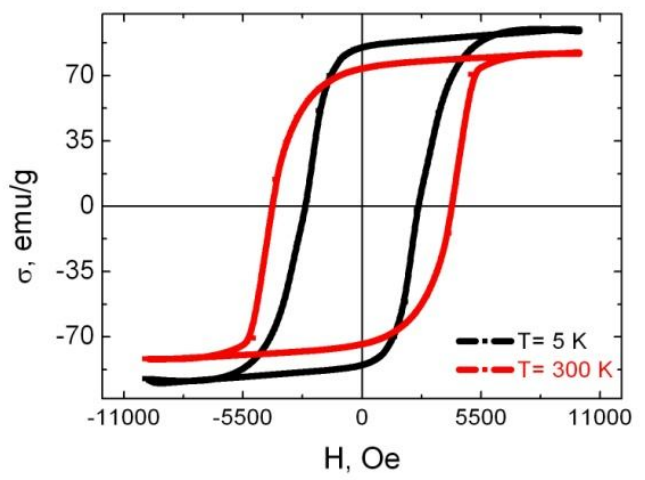

Figure 2. The field dependences of the specific magnetization $\sigma(\mathrm{H})$ of the $\mathrm{SrFe}_{11.9} \mathrm{In}_{0.1} \mathrm{O}_{19}$ compound measured at 5 (black line) and 300 (red line) K.

Fig. 2 shows the unusual behavior of the coercive force $\left(\mathrm{H}_{\mathrm{c}}\right)$ of the $\mathrm{SrFe}_{11.9} \mathrm{In}_{0.1} \mathrm{O}_{19}$ compound in comparison, for instance, with $\mathrm{Fe}_{3} \mathrm{O}_{4}$ [25] or $\mathrm{Bi}_{1-x} \mathrm{La}_{\mathrm{x}} \mathrm{FeO}_{3}$ [26], where the coercive force increases as the temperature is decreased. In this study, the magnitude $\mathrm{H}_{\mathrm{c}}$ decreases in $\sim 1.6$ times from $\mathrm{H}_{\mathrm{c}}$ $\sim 4185$ Oe to $\mathrm{H}_{\mathrm{c}} \sim 2560 \mathrm{Oe}$, when the ambient temperature is decreased from 300 to $5 \mathrm{~K}$. A decrease of coercivity with decreasing temperature was previously observed in $\mathrm{SrFe}_{12} \mathrm{O}_{19}$ [27] and $\mathrm{BaFe}_{12} \mathrm{O}_{19}$ [28] ferrites. The similar behavior was explained by the imperfection of the crystal structure due to its proximity to the amorphous state. In our case the sample $\mathrm{SrFe}_{11.9} \mathrm{In}_{0.1} \mathrm{O}_{19}$ was annealed at $1300^{\circ} \mathrm{C}(6 \mathrm{~h})$. This fact and the high quality of X-ray pattern (see below) confirm the good state of the crystal structure. Earlier studies of ferromagnets [20] revealed a significant increase in their coercive force during the transition from a multi-domain to a single-domain state. In addition, according to Weiss's theory, the magnetization in ferro- or ferrimagnetics is accompanied by the growth of some domains at the expense of others. Therefore, in this case, it can be assumed that the multidomain crystallite has less Bloch walls with increasing temperature due to growing domain size with temperature.

\subsection{Ferroelectric properties}

The electric voltage dependence of the polarization $\mathrm{P}$ for strontium hexaferrite $\mathrm{SrFe}_{11.9} \mathrm{In}_{0.1} \mathrm{O}_{19}$ measured at room temperature is shown in Fig.3. A rapid increase in the saturation polarization of strontium ceramic was observed under electric field. The presence of hysteresis loops and the appearance of remanent electrical polarization at room temperature, which can be reversed applying an external electric field, allows to characterize strontium ferrite as ferroelectric. The maximal values of polarization $\left(\mathrm{P}_{\max }\right)$ and spontaneous polarization $\left(\mathrm{P}_{\mathrm{r}}\right)$ as well as the coercive force $\left(E_{C}\right)$ determined from the hysteresis loops have the following values: $4.17 \mathrm{nC} / \mathrm{cm}^{2}, 1.35 \mathrm{nC} / \mathrm{cm}^{2}$ and $138 \mathrm{~V}$, respectively.

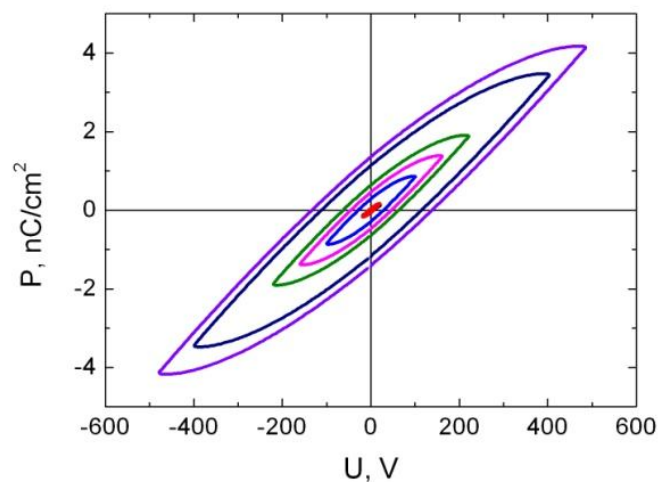

Figure 3. Ferroelectric hysteresis loops of $\mathrm{SrFe}_{11.9} \mathrm{In}_{0.1} \mathrm{O}_{19}$ ceramic.

Ferroelectric properties were earlier discovered in the initial compound $\mathrm{SrFe}_{12} \mathrm{O}_{19}$ [5], nevertheless, their occurrence mechanism in the solid solutions of hexaferrites remains controversial. In particular, the main reason of the generation of ferroelectricity in the initial compounds $\mathrm{AFe}_{12} \mathrm{O}_{19}$ (where $\mathrm{A}=\mathrm{Pb}, \mathrm{Ba}, \mathrm{Sr}$ ) [4 - 6] was suggested the distortion of one of the oxygen octahedral. Meanwhile, in Ref. [8] the main reason of the ferroelectric properties in solid solutions was called the occurrence of a non-collinear magnetic structure.

One more explanation of the appearance of spontaneous polarization in solid solutions of barium ferrites was proposed in previously published Ref. [29 - 31], where ferroelectricity is a consequence of non-centrosymmetric distortion of the unit cell. The discussion of possible reasons of the occurrence of ferroelectric properties in the $\mathrm{SrFe}_{11.9} \mathrm{In}_{0.1} \mathrm{O}_{19}$ compound will be given below.

\subsection{Crystal structure}

According to X-ray diffraction data, the $\mathrm{SrFe}_{11.9} \mathrm{In}_{0.1} \mathrm{O}_{19}$ sample is homogeneous. As was mentioned above, the crystal structure of strontium hexaferrite is well described by the centrosymmetric space group $\mathrm{P}_{3} / \mathrm{mmc}$ (No. 194) and correspond ICDD card 00-033-1340. The average crystallite size $<\mathrm{D}>\sim 1180 \AA$ was determined from the broadening of the diffraction peak (0211) by Scherrer's equation (1). Figure 4 shows a general view of the X-ray pattern of the $\mathrm{SrFe}_{11.9} \mathrm{In}_{0.1} \mathrm{O}_{19}$ compound, which crystal structure was refined by the Rietveld method in the framework of the space group $\mathrm{P}_{3} / \mathrm{mmc}$. However, the description of the crystal structure with the centrosymmetric $\mathrm{SG} \mathrm{P}_{3} / \mathrm{mmc}$ means that the unit cell has an inversion center, which contradicts the appearance of ferroelectric properties in the $\mathrm{SrFe}_{11.9} \mathrm{In}_{0.1} \mathrm{O}_{19}$ ferrite shown in Fig.3.

It was proposed to consider the crystal structure of hexaferrites in the framework of non-centrosymmetric SG $\mathrm{P}_{3} \mathrm{mc}$ (No. 186), according to Ref. [29]. However, no 
information was given about structural parameters such as unit cell parameters, atomic coordinates, etc.

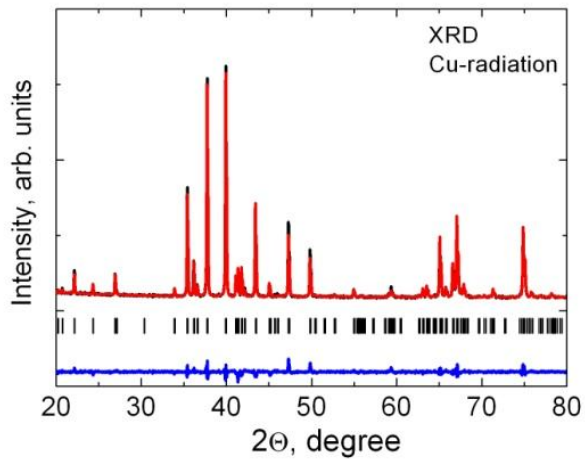

Figure 4. The general view of X-ray pattern of $\mathrm{SrFe}_{11.9} \mathrm{In}_{0.1} \mathrm{O}_{19}$ ferrite refined with the Rietveld method in the framework of the generally accepted centrosymmetric space group $\mathrm{P}_{3} / \mathrm{mmc}$ (No. 194).

Therefore, in order to clarify the real crystal structure of strontium ferrite, neutron diffraction patterns were measured in a wide temperature range from 1.5 to $350 \mathrm{~K}$. The general view of neutron diffraction patterns is shown in Fig.5. The temperature dependencies of the crystal structure parameters $(a$ and $c$ ) and the volume of the unit cell $(\mathrm{V})$ are shown in Fig.6. The area, where thermal extension of lattice parameters is absent, was observed in the low-temperature region. For the first time, such behavior was discovered in solid solutions of barium hexaferrite [32]. There is a difference in the temperature dependencies of the lattice parameters $a$ and $c$.

In particular, $c$ parameter has insignificant variation in the temperature range from 1.5 to $50 \mathrm{~K}$, while $a$ parameter practically does not change in wider temperature area from 1.5 to $150 \mathrm{~K}$. This difference in the changes of the unit cell parameters $a$ and $c$ can be related with strong magneto crystalline anisotropy of strontium hexaferrite. While the presence of a temperature range, where thermal expansion is absent, can be explained by mutual rotations and tilts of the oxygen polyhedra, similarly to Ref.[32].

The refinement of the crystal structure of the $\mathrm{SrFe}_{11.9} \mathrm{In}_{0.1} \mathrm{O}_{19}$ compound was carried out within both the centrosymmetric $\mathrm{P} 6_{3} / \mathrm{mmc}$ (No. 194) and noncentrosymmetric $\mathrm{P}_{3} \mathrm{mc}$ (No. 186) space groups. The results of the refinement are shown in Tables 1 and 2 .

The comparison of the relevance factors $\left(\mathrm{R}_{\mathrm{exp}}, \mathrm{R}_{\mathrm{wp}}, \chi^{2}\right)$ from Table 1 and 2 and difference curves, normalized to the statistical errors from Fig. 5, indicates that the crystal structure of strontium hexaferrite can be described quite well by both the centrosymmetric and noncentrosymmetric space group in a wide temperature range. However, figures of merit for non- centrosymmetric space group have slightly lower values than for the centrosymmetric space group (see Table 1 and 2). Therefore, observation of ferroelecric properties in the $\mathrm{SrFe}_{11.9} \operatorname{In}_{0.1} \mathrm{O}_{19}$ compound shown in Fig.3 and less magnitudes figures of merit in the case of the non- centrosymmetric space group (see Table 2) allows us to give preference to SG $\mathrm{P}_{3} \mathrm{mc}$ (No. 186).

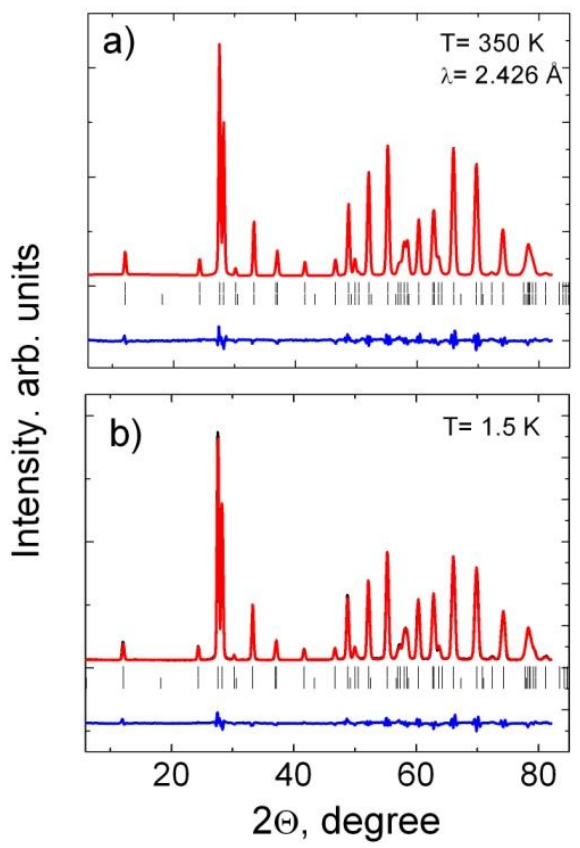

Figure 5. Neutron diffraction patterns of $\mathrm{SrFe}_{11.9} \mathrm{In}_{0.1} \mathrm{O}_{19}$ compound measured on diffractometer G 4-1 at temperatures 350 - (a) and $1.5 \mathrm{~K}$ - (b) and processed by the Rietveld method. Experimental (black curve) and calculated (red curve) curves, as well as the difference function normalized to the statistical error (blue curve) are shown. Vertical bars indicate the calculated positions of the diffraction peaks for the crystalline (top) and magnetic (bottom) structures of strontium hexaferrite, calculated in the framework of the non-centrosymmetric SG $\mathrm{P}_{3} \mathrm{mc}$ (No. 186).

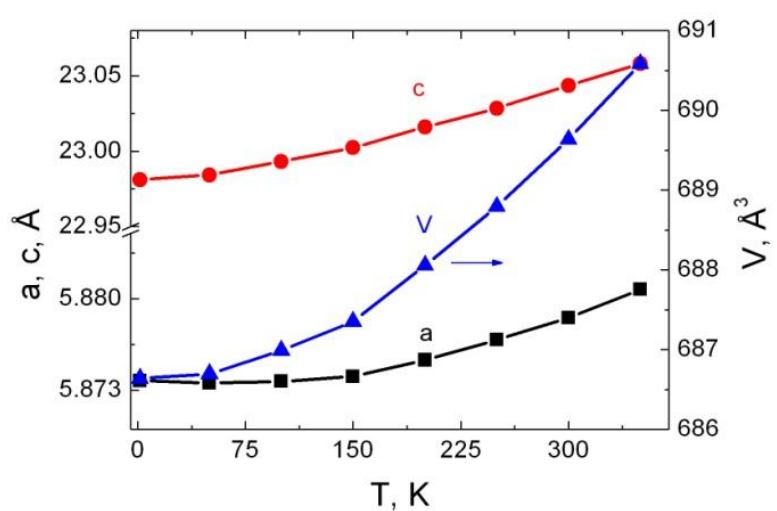

Figure 6. Temperature dependence of structural parameters $a$ and $c$ (left scale) and volume $\mathrm{V}$ (right scale) of unit cell for $\mathrm{SrFe}_{1.9} \mathrm{In}_{0.1} \mathrm{O}_{19}$ compound.

Fragments of the crystal structure of unit cells for centrosymmetric and non-centrosymmetric space groups are 
shown in Fig.7, a - e. In spite of the completely equal values of lattice parameters for unit cells both space groups (see Fig. 6 and Tables 1 and 2), there is a difference in the distortion of individual structural elements.

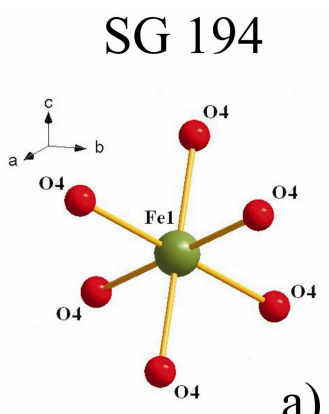

a)

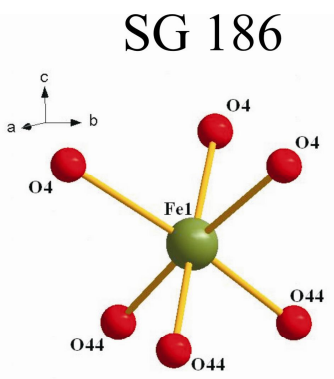

b)
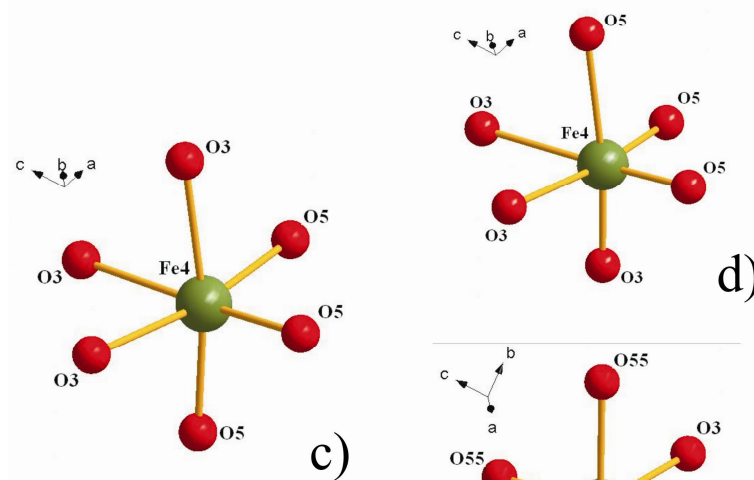

d)

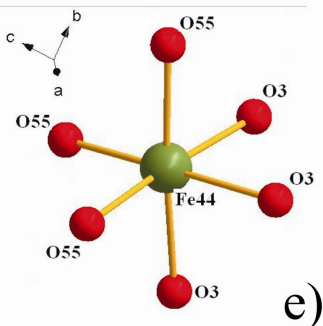

e)

Figure 7. Fragments of unit cell: oxygen octahedron around Fe1 ion - undistorted for SG 194 (a) and distorted for SG 186; and oxygen octahedra around Fe4/Fe44 for SG 194 (c) and for SG 186 (d and e). (Oxygen ions (O) are designed by red spheres and iron ions $(\mathrm{Fe})$ are designed by dark green spheres.)

To evaluate the distortion of the oxygen octahedron $\left(\mathrm{S}_{\mathrm{BO}}\right)$, the equation was used:

$$
S_{\mathrm{FeO}_{6}}(T)=\frac{100}{\langle d(T)\rangle} \times \sqrt{\frac{\sum_{i=1}^{6}\left(d_{i}(T)-\langle d(T)\rangle\right)^{2}}{5}}
$$

where $\mathrm{d}_{\mathrm{i}}(\mathrm{T})$ is the distance between iron ions and the nearest oxygen ions; $<\mathrm{d}(\mathrm{T})>$ is the average $<\mathrm{Fe}-\mathrm{O}>$ distance in the oxygen octahedron.

The distortions of the oxygen octahedron at different ambient temperatures are shown in Fig. $8 \mathrm{a}$ and $\mathrm{b}$.
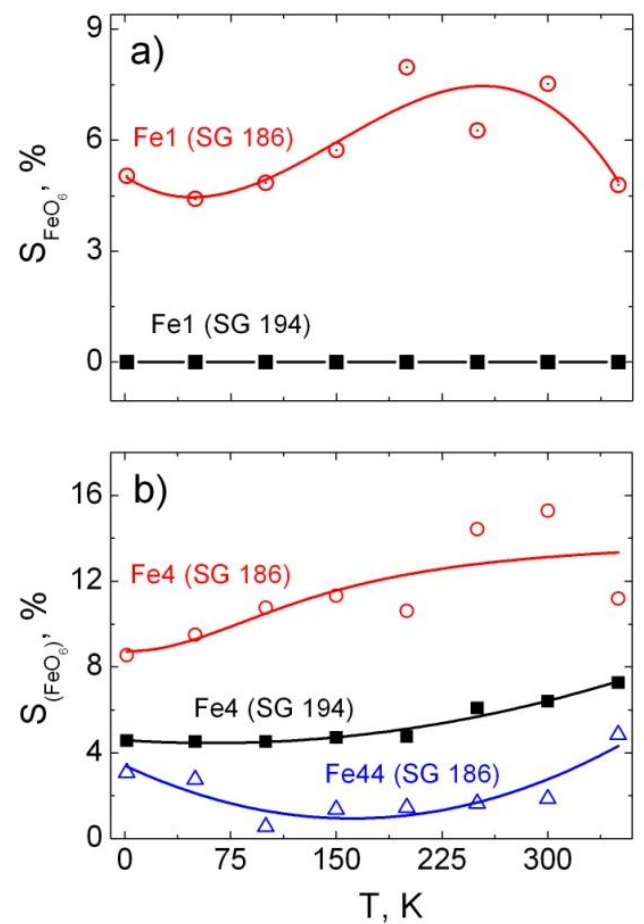

Figure 8. Temperature dependencies of distortions of oxygen octahedra around: Fe1 ion - (a); and Fe4 and Fe44 ions - (b). (Oxygen octahedra of unit cell for SG 194 are designated with solid squares, and for SG 186 - with empty circles and triangles.)

According to Fig.8, in the case of the description of the unit cell of the $\mathrm{SrFe}_{11.9} \operatorname{In}_{0.1} \mathrm{O}_{19}$ compound with the centrosymmetric space group No. 194, the oxygen octahedra of $\mathrm{Fel}$ ions located in crystallographic positions (2a) have no distortions in the temperature range from 1.5 to $350 \mathrm{~K}$. In the case of a unit cell description in the framework of the noncentrosymmetric space group (No. 186), the distortions of a similar oxygen octahedron achieve $\sim 5-7 \%$ (see Fig. 8 a) in the studied temperature range.

Another behavior is observed in oxygen surroundings of $\mathrm{Fe}_{4}$ ion (see Fig. 7, c and d). In the case of centrosymmetric SG No. 194, distortions of the oxygen octahedra that surround crystallographic positions $\left(4 \mathrm{f}_{\mathrm{VI}}\right)$ monotonously increase from $\sim 4.6$ to $7.3 \%$ as the ambient temperature is increased up to $350 \mathrm{~K}$ (see Fig. $8 \mathrm{~b}$ ). In the case of a noncentrosymmetric SG 186, there is a difference in the distortion of neighboring oxygen octahedra (see Fig. 8 b). It should be noted that the distortion of oxygen polyhedra can be a reason of the appearance of local electric dipoles for both centrosymmetric and non-centrosymmetric space groups. However, unequal distortions of neighboring oxygen octahedra are the main reason of uncompensated local dipole 
moments that leads to the appearance of ferroelectric properties. The influence of the temperature factor on the magnitude of local electric dipoles is shown in Fig. 9 a and b.

Therefore, taking into account the presence of an inversion center in the centrosymmetric unit cell, there will be no spontaneous polarization due to the compensation of local electric dipoles. On the contrary, in the noncentrosymmetric unit cell, unequal distortion of neighboring oxygen surroundings (see Fig. $8 \mathrm{a}$ and $\mathrm{b}$ ) is the only cause of the occurrence of spontaneous polarization.
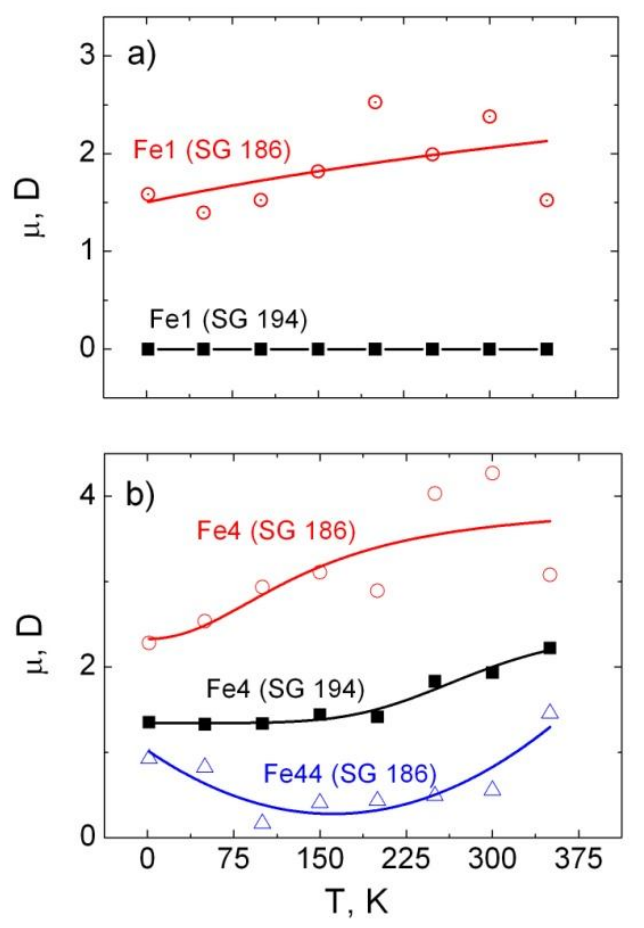

Figure 9. Temperature dependencies of electric dipoles as result of distortions of oxygen octahedrons around: Fe1 (a); and Fe4 and Fe44 - (b). (Electric dipoles of unit cell for SG 194 are designated with solid squares, and for SG 186 with empty circles and triangles.)

\subsection{Magnetic structure}

As was mentioned above, the main feature of the neutron diffraction method is a direct study of magnetic structure materials. Neutron diffraction patterns were measured in a wide temperature range from 1.5 to $350 \mathrm{~K}$, which is well below the paramagnetic - ferrimagnetic phase transition temperature $\left(\mathrm{T}_{\mathrm{c}} \sim 670 \mathrm{~K}\right)$ for a given compound (see Fig. 1). In Fig.5, the bottom row of vertical bars indicates the positions of diffraction peaks calculated by the Rietveld method for the magnetic structure of $\mathrm{SrFe}_{11.9} \mathrm{In}_{0.1} \mathrm{O}_{19}$ hexaferrite. The absence of additional magnetic peaks on neutron diffraction patterns indicates that the wave vector of this ferrimagnetic structure: $\mathbf{k}=[0,0,0]$.

The magnetic structure of $\mathrm{SrFe}_{11.9} \mathrm{In}_{0.1} \mathrm{O}_{19}$ ferrite satisfies to the model proposed by Gorter [33]. It has ferrimagnetic order, where all magnetic moments of $\mathrm{Fe}^{3+}$ ions are directed along $c$ axis, which is easy magnetization axis. This ferrimagnetic structure is formed by two ferromagnetic sublattices that directed in the opposite direction. The magnetic moments of $\mathrm{Fe}^{3+}$ ions located in octahedral (2a and $12 \mathrm{k}$ ) and bipyramidal (2b) oxygen surroundings are oriented in opposite to the magnetic moments of iron ions occupying positions $\left(4 \mathrm{f}_{\mathrm{IV}}\right.$ and $\left.4 \mathrm{f}_{\mathrm{VI}}\right)$.

As was mentioned above the unit cell was refined in the framework both centrosymmetric $\left(\mathrm{P}_{3} / \mathrm{mmc}\right)$ and noncentrosymmetric $\left(\mathrm{P}_{3} \mathrm{mc}\right)$ space groups therefore magnetic moments were also calculated separately for each space group. The temperature dependencies of magnetic moments of $\mathrm{Fe}^{3+}$ ions in various crystallographic positions are shown in Fig. $10 \mathrm{a}$ and b. Values of magnetic moments at different crystallographic positions in the entire temperature range do not exceed $5 \mu_{\mathrm{B}}$, which is characteristic for the magnetic moment of $\mathrm{Fe}^{3+}$. It is an indirect confirmation of the correctness of the calculations.

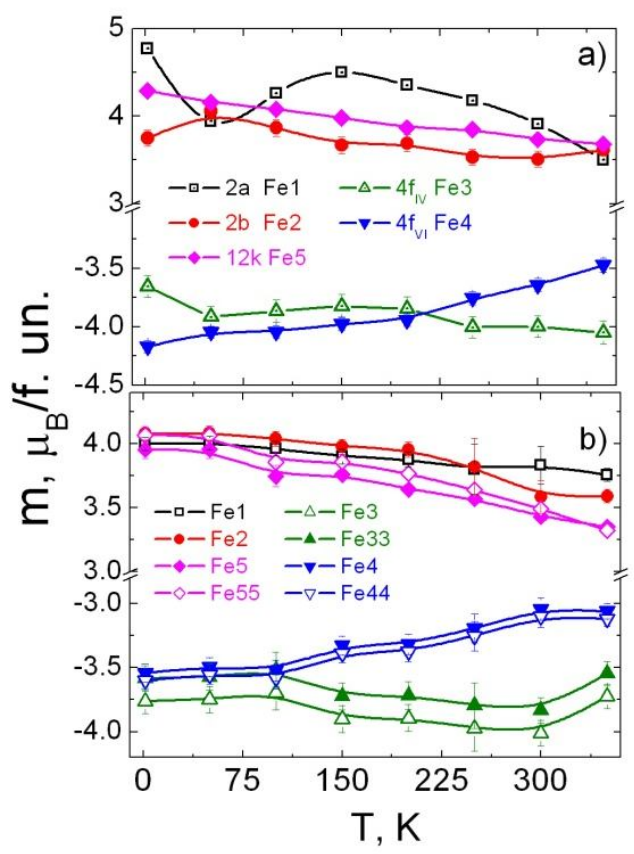

Figure 10. Temperature dependencies of local magnetic moments for suitable crystallographic positions for SG $194-$ (a) and SG 186 - (b). Designations in the figures are follow: Fe1 - 口; Fe2 - •; Fe3 - $\Delta$; Fe33 - $\boldsymbol{\Delta} ;$ Fe4 - $\boldsymbol{\nabla} ;$ Fe44 - $\nabla$; Fe5 - $\triangleleft$ Fe55 - $\diamond$. 
The total magnetic moment per formula unit of strontium ferrite $\left(\mathrm{SrFe}_{12} \mathrm{O}_{19}\right)$ with a centrosymmetric structure can be calculated with equation [18]:

$$
\begin{aligned}
& M_{\text {total }}(T)=1\left[m_{F e 1}(T)\right]+1\left[m_{F e 2}(T)\right]- \\
& 2\left[m_{F e 3}(T)\right]-2\left[m_{F e 4}(T)\right]+6\left[m_{F e 5}(T)\right]
\end{aligned}
$$

In the case of a non- centrosymmetric structure, the total magnetic moment was calculated as:

$$
M_{\text {total }}(T)=1\left[m_{F e 1}(T)\right]+1\left[m_{F e 2}(T)\right]-
$$$$
1\left[m_{\mathrm{Fe} 3}(T)\right]-1\left[m_{\mathrm{Fe} 33}(T)\right]-1\left[m_{\mathrm{Fe} 4}(T)\right]-
$$$$
1\left[m_{\mathrm{Fe} 44}(T)\right]+3\left[m_{\mathrm{Fe} 5}(T)\right]+3\left[m_{\mathrm{Fe} 55}(T)\right] \text { (7) }
$$

where $\mathrm{m}_{\mathrm{i}}$ is the magnetic moment of $\mathrm{Fe}^{3+}$ ion in the $i$-th sublattice.

Temperature dependencies of the total magnetic moments $\left(\mathrm{M}_{\text {total }}\right)$ are shown in Fig. 11. It should be noted that the

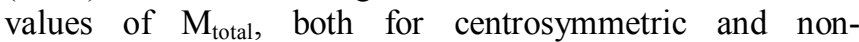
centrosymmetric structures, have insignificant difference from each other, which does not allow giving the preference to one of them. Moreover, the general view of the magnetic structure is comparable both for SG 194 and SG 186 and is shown in the insert Fig. 11.

The magnitude of the total magnetic moment has slightly less value than $20 \mu_{\mathrm{B}}$ per formula unit (f.un.) in the lowtemperature region. It is due to the influence of diamagnetic In ions, which disrupts the exchange interaction between neighboring $\mathrm{Fe}^{3+}-\mathrm{O}-\mathrm{Fe}^{3+}$ ions. The decrease of total magnetic moment is observed with increasing of the ambient temperature. Such behavior is the result of magnetic order disrupting due to an increase in thermal oscillations of the ions forming the crystal lattice.

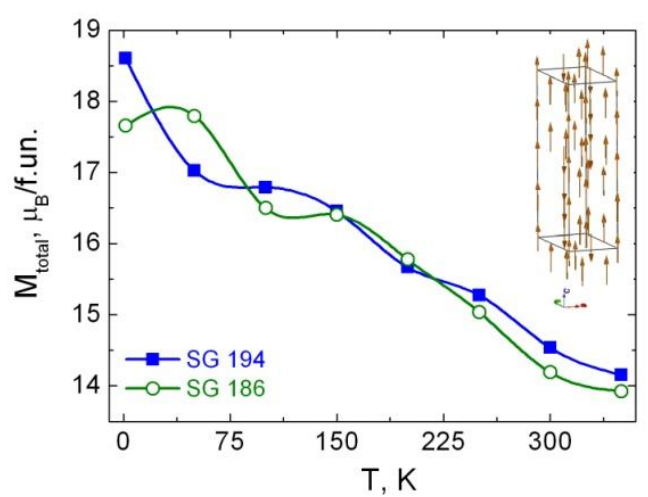

Figure 11. Temperature dependencies of total magnetic moments for SG 194 - (a) and SG 186 - (b). In inset: general view of magnetic structure of $\mathrm{SrFe}_{11.9} \mathrm{In}_{0.1} \mathrm{O}_{19}$ compound is shown.

\section{Conclusions}

The presence of spontaneous polarization at room temperature was observed in strontium ferrite partially substituted with diamagnetic In ions. The appearance of spontaneous polarization in strontium hexaferrite indicates its ferroelectric property that contradicts to the generally accepted description of the crystal structure of similar materials with centrosymmetric $\mathrm{SG} \mathrm{P}_{3} / \mathrm{mmc}$. The discrepancy between FC and ZFC temperature dependencies of magnetizations indicates the presence of a magnetically inhomogeneous system. It was found that the coercive field decreased in $\sim 1.6$ times as the ambient temperature increased from 5 to $300 \mathrm{~K}$, which may be the result of growing domain size with temperature. The fact that the ferroelectric properties of the $\mathrm{SrFe}_{11.9} \mathrm{In}_{0.1} \mathrm{O}_{19}$ compound coexist with ferrimagnetic ordering and its high Curie temperature $\left(\mathrm{T}_{\mathrm{c}} \sim\right.$ $670 \mathrm{~K}$ ) allows to consider this material as a possible multiferroic.

The crystal and magnetic structures of the $\mathrm{SrFe}_{11.9} \mathrm{In}_{0.1} \mathrm{O}_{19}$ compound was studied by the neutron diffraction method in a wide temperature range from 1.5 to $350 \mathrm{~K}$. Insignificant changes of the lattice parameters were observed in strontium hexaferrites in the temperature range from 1.5 to 150 . It was explained by mutual rotations and tilts of oxygen surroundings.

In order to determine the real type of the crystal structure of strontium ferrite, it was refined within the framework both centrosymmetric $\mathrm{P}_{3} / \mathrm{mmc}$ (No. 194) and noncentrosymmetric $\mathrm{P}_{3} \mathrm{mc}$ (No. 186) space groups. It was shown that the crystal structure of the $\mathrm{SrFe}_{11.9} \mathrm{In}_{0.1} \mathrm{O}_{19}$ compound can be described quite well by both space groups. However, less values figures of merit $\left(\mathrm{R}_{\exp }, \mathrm{R}_{\mathrm{wp}}\right.$ and $\left.\chi^{2}\right)$ and the presence of ferroelectric properties allows to give preference to the non-centrosymmetric $\mathrm{P}_{3} \mathrm{mc}$ (No. 186) space group. Moreover, using the non-centrosymmetric $\mathrm{P}_{3} \mathrm{mc}$ (No. 186) space group for describing crystal structure of the $\mathrm{SrFe}_{11.9} \mathrm{In}_{0.1} \mathrm{O}_{19}$ allows to explain the appearance of spontaneous polarization as a result of unequal distortions of neighboring oxygen surroundings because of absence inversion center in the unit cell.

The magnetic structure of the $\mathrm{SrFe}_{11.9} \mathrm{In}_{0.1} \mathrm{O}_{19}$ compound is well described by Gorter's model, according to which magnetic moments of all $\mathrm{Fe}^{3+}$ ions are ordered along hexagonal $c$ axis. The values of the total and local magnetic moments in various crystallographic positions in a wide temperature range were determined for unit cell described within the framework of both centrosymmetric $\mathrm{P} 6_{3} / \mathrm{mmc}$ (No. 194) and non-centrosymmetric $\mathrm{P}_{3} \mathrm{mc}$ (No. 186) space groups.

\section{Acknowledgements}

This project has received funding from the EU - H2020 research and innovation programme (support of the scientific infrastructure and scientific mobility for measurements in EU countries) under grant agreement No 654360 having benefitted from the access provided by CSIC/ICMAB in Barcelona (ES) and by CEA/LLB in Paris (FR) within the framework of the NFFA - Europe Transnational Access Activity. The work was support by the Russian Science Foundation (Agreement No. 19-19-00694 of 06 May 2019). 


\section{References}

[1] Tsutaoka T and Koga N, 2013 Magnetic phase transitions in substituted barium ferrites $\mathrm{BaFe}_{12-\mathrm{x}}\left(\mathrm{Ti}_{0.5} \mathrm{Co}_{0.5}\right)_{\mathrm{x}} \mathrm{O}_{19}(\mathrm{x}=0.5) J$. Magn. Magn. Mater. 32536 - 41

[2] Shirk B T and Buessem W R, 1969 Temperature Dependence of $\mathrm{M}_{\mathrm{s}}$ and $\mathrm{K}_{1}$ of $\mathrm{BaFe}_{12} \mathrm{O}_{19}$ and $\mathrm{SrFe}_{12} \mathrm{O}_{19}$ Single Crystals J. Appl. Phys. $401294-1296$

[3] Adelskold V, 1938 X-ray studies on magneto-plumbite, $\mathrm{PbO}$ $* 6 \mathrm{Fe}_{2} \mathrm{O}_{3}$ and other substances resembling beta-alumina, $\mathrm{Na}_{2} \mathrm{O}$ $11 \mathrm{Al}_{2} \mathrm{O}_{3}$ Arkiv for Kemi Mineralogioch Geologi A 12 1-9

[4] Tann G and Chen X, 2013 Structure and multiferroic properties of barium hexaferrite ceramics J. Magn. Magn. Mater. 327 8790

[5] Tann G and Chen X, 2013 Synthesis, Structures, and Multiferroic Properties of Strontium Hexaferrite Ceramics // J. Electr. Mat. 42 906-911

[6] Tan G and Wang M 2011 Multiferroic $\mathrm{PbFe}_{12} \mathrm{O}_{19}$ Ceramics $J$. Electrocer. 26170 - 174

[7] Kostishyn V G, Panina L V, Timofeev A V, Kozhitov L V, Kovalev A N, and Zyuzin A K 2016 Dual ferroic properties of hexagonal ferrite ceramics $\mathrm{BaFe}_{12} \mathrm{O}_{19}$ and $\mathrm{SrFe}_{12} \mathrm{O}_{19} J$. Magn. Magn. Mater. $400327-332$

[8] Tokunaga Y, Kaneko Y, Okuyama D, Ishiwata S, Arima T, Wakimoto S, Kakurai K, Taguchi Y and Tokura Y 2010 Multiferroic M-Type Hexaferrites with a Room-Temperature Conical State and Magnetically Controllable Spin Helicity // Phys. Rev. Lett. 105 257201(1-4)

[9] Turchenko V, Trukhanov A, Trukhanov S, Balasoiu M, Lupu N 2019 Correlation of crystalline and magnetic structures of barium ferrites with dual ferroic properties // J. Magn. Magn. Mater. 477 9-16

[10] Turchenko V. A., Balagurov A. M., Trukhanov S. V., Trukhano A. V. 2019 Refinement of the Atomic and Magnetic Structures of Solid Solutions $\mathrm{BaFe}_{12-\mathrm{x}} \mathrm{In}_{\mathrm{x}} \mathrm{O}_{19}(\mathrm{x}=0.1-1.2)$ by the Neutron Diffraction Method // J. Surf. Investig.: X-ray, Synchrotron and Neutron Techniques, 13 69-81.

[11] Trukhanov A.V., Darwish M.A., Panina L.V., Morchenko A.T., Kostishyn V.G., Turchenko V.A., Vinnik D.A., Trukhanova E.L., Astapovich K.A., Kozlovskiy A.L., Zdorovets M., Trukhanov S.V. 2019 Features of crystal and magnetic structure of the $\mathrm{BaFe}_{12-\mathrm{x}} \mathrm{Ga}_{\mathrm{x}} \mathrm{O}_{19}(\mathrm{x} \leq 2)$ in the wide temperature range // J. All. Comp. 791 522-529.

[12] Turchenko V.A., Trukhanov S.V., Balagurov A.M., Kostishyn V.G., Trukhanov A.V., Panina L.V., Trukhanova E.L. 2018 Features of crystal structure and dual ferroic properties of $\mathrm{BaFe}_{12-x} \mathrm{Me}_{x} \mathrm{O}_{19}\left(\mathrm{Me}=\mathrm{In}^{3+}\right.$ and $\left.\mathrm{Ga}^{3+} ; \mathrm{x}=0.1-1.2\right)$ // J. Magn. Magn. Mater. 464 139-147.

[13] Trukhanov S.V., Trukhanov A.V., Turchenko V.A., Trukhanov An.V., Tishkevich D.I., Trukhanova E.L., Zubar T.I., Karpinsky D.V., Kostishyn V.G., Panina L.V., Vinnik D.A., Gudkova S.A., Trofimov E.A., Thakur P., Thakur A., Yang Y. 2018 Magnetic and dipole moments in indium doped barium hexaferrites // J. Magn. Magn. Mater. 457 83-96.

[14] Pullar R C 2012 Hexagonal ferrites: A review of the synthesis, properties and applications of hexaferrite ceramics // Progr. Mater. Science 57 1191-1334

[15] Ghimire M, Yoon S, Wang L, Neupane D, Alam J, Mishra S R 2018 Influence of La content on magnetic properties of $\mathrm{Cu}$ doped M-type strontium hexaferrite: Structural, magnetic, and Mossbauer spectroscopy study J. Magn. Magn. Mater. 454 110-120
[16] Kikuchi T, Nakamura T, Yamasaki T, Nakanishi M, Fujii T, Takada J, Ikeda Y 2010 Magnetic properties of La-Co substituted M-type strontium hexaferrites prepared by polymerizable complex method J. Magn. Magn. Mater. 322 2381-2385

[17] Chantrell R W, Walmsley N S, Gore J and Maylin M 1999 Theoretical studies of the field-cooled and zero-field cooled magnetization of interacting fine particles J. Appl. Phys. 854340 - 4343

[18] Binder K, Young A P 1986 Spin glasses: experimental facts, theoretical concepts, and open questions Rev. Modern Phys. 58 802-976.

[19] Coffey W T, Crothers D S F, Dormann J L, Geoghegan L J, Kalmykov Yu P, Waldron J T and Wickstead A W 1995 The effect of an oblique magnetic field on the superparamagnetic relaxation time // J. Magn. Magn. Mater. 145 L263-L267

[20] Kneller E F and Luborsky F E 1963 Particle Size Dependence of Coercivity and Remanence of Single-Domain Particles J. Appl. Phys. 34 656-658

[21] Ajroudi L, Mliki N, Bessans L, Madigou V, Villain S and Leroux C 2014 Magnetic, electric and thermal properties of cobalt ferrite nanoparticles Mater. Research Bulletin, Elsevier 5949

[22] Bean C P and Livingston J D 1959 Superparamagnetism J. Appl. Phys. $30 \mathrm{~S} 120$ - S129

[23] Smit J and Wijn H P 1959 Ferrites: physical properties of ferrimagnetic oxides in relation to their technical application (Wiley-Inter Science, New York)

[24] Gubin S P, Koksharov Yu A, Khomutov G B and Yurkov G Yu 2005 Magnetic nanoparticles: preparation, structure and properties Russ. Chem. Rev. 74 489-520

[25] Wang J, Beeli P, Meng L H and Zhao G M 2011 Hightemperature magnetic properties of noninteracting randomly oriented single-domain $\mathrm{Fe}_{3-\delta} \mathrm{O}_{4}$ nanoparticles Phys. Lett. A 375 $1510-1513$

[26] Pashchenko A V, Liedienov N A, Li Q, Tatarchuk D D, Turchenko V A, Makoed I I, Sycheva V Ya, Voznyak A V, Kladko V P, Gudimenko A I, Didenko Y V, Kozakov A T and Levchenko G G 2019 Structure, non-stoichiometry, valence of ions, dielectric and magnetic properties of single-phase $\mathrm{Bi}_{0.9} \mathrm{La}_{0.1} \mathrm{FeO}_{3-\delta}$ multiferroics // J. Magn. Magn. Mater. 483 100-113

[27] Sivakumar P, Shani L, Yeshurun Y, Shaulov A and Gedanken A 2016 Facile sonochemical preparation and magnetic properties of strontium hexaferrite $\left(\mathrm{SrFe}_{12} \mathrm{O}_{19}\right)$ nanoparticles // J. Mater. Sci: Mater. Electron $271-8$

[28] Pashkova E V, Solovyova E D, Kolodiazhnyi T V, Ivanitskii V P, Belous A G 2014 Effect of heat treatment on the phase composition, structure and magnetic properties of M-type barium hexaferrite // J. Magn. Magn. Mater. 3681 - 7

[29] Mikheykin A S, Zhukova E S, Torgashev V I, Razumnaya A G, Yuzyuk Y I, Gorshunov B P, Prokhorov A S, Sashin A E, Bush A A and Dressel M 2014 Lattice anharmonicity and polar soft mode in ferrimagnetic M-type hexaferrite $\mathrm{BaFe}_{12} \mathrm{O}_{19}$ single crystal Eur. Phys. J. B 87232 (1-9)

[30] Trukhanov A.V., Trukhanov S.V., Panina L.V., Kostishyn V.G., Kazakevich I.S., Trukhanov An.V., Trukhanova E.L., Natarov V.O., Turchenko V.A., Salem M.M., Balagurov A.M. 2017 Evolution of structure and magnetic properties for $\mathrm{BaFe}_{11.9} \mathrm{Al}_{0.1} \mathrm{O}_{19}$ hexaferrite in a wide temperature range // $J$. Magn. Magn. Mater. 426 487-496

[31] Trukhanov S V, Trukhanov A V, Turchenko V A, Trukhanov An V, Trukhanova E L, Tishkevich D I, Ivanov V M, Zubar T I, Salem M, Kostishyn V G, Panina L V, Vinnik D 
A, Gudkova S A 2018 Polarization origin and iron positions in indium doped barium hexaferrites Ceram. Intern. 44 290-300

[32]

Turchenko V, Trukhanov A, Trukhanov S, Bobrikov I and Balagurov A M 2016 Features of crystal and magnetic structures of solid solutions $\mathrm{BaFe}_{12-\mathrm{x}} \mathrm{D}_{\mathrm{x}} \mathrm{O}_{19}\left(\mathrm{D}=\mathrm{Al}^{3+}, \mathrm{In}^{3+} ; \mathrm{x}=\right.$ $0.1)$ in a wide temperature range Eur. Phys. J. Plus 131 82(110)
[33] Gorter E W 1957 Saturation magnetization of some ferrimagnetic oxides with hexagonal crystal structures Proc IEEE B: Radio and Electr. Eng. 104255 - 260 
Table 1.

Crystal structure parameters, figures of merit and atomic positions refined in the framework of centrocymmetric SG P $6_{3} / \mathrm{mmc}$ (№ 194) with the Rietveld method for different temperatures. Atoms are located in positions: $\operatorname{Sr}(2 \mathrm{~d})(2 / 3,1 / 3,1 / 4)$, Fe1 (2a) $(0,0,0)$; Fe2 (2b) $(0,0,1 / 4)$; $(\mathrm{Fe} / \mathrm{In}) 3\left(4 \mathrm{f}_{\mathrm{IV}}\right)(1 / 3,2 / 3, z) ;(\mathrm{Fe} / \mathrm{In}) 4\left(4 \mathrm{f}_{\mathrm{VI}}\right)(1 / 3,2 / 3, z) ;(\mathrm{Fe} / \mathrm{In}) 5(12 \mathrm{k})(x, 2 x, z) ; \mathrm{O} 1(4 \mathrm{e})(0,0$, $z)$; O2 (4f) $(1 / 3,2 / 3, z)$; O3 (6h) $(x, 2 x, 1 / 4)$; O4 (12k) $(x, 2 x, z)$; O5 (12k) $(x, 2 x, z)$.

\begin{tabular}{|c|c|c|c|c|}
\hline $\begin{array}{l}\text { Atomic } \\
\text { positions }\end{array}$ & $50 \mathrm{~K}$ & $150 \mathrm{~K}$ & $250 \mathrm{~K}$ & $350 \mathrm{~K}$ \\
\hline$a,(\AA)$ & $5.8736(1)$ & $5.8741(2)$ & $5.8769(2)$ & $5.8807(2)$ \\
\hline$c,(\AA)$ & $22.9843(6)$ & $23.0023(7)$ & $23.0285(7)$ & $23.0581(8)$ \\
\hline$V,\left(\AA^{3}\right)$ & $686.69(3)$ & $687.35(3)$ & $688.79(4)$ & $690.58(4)$ \\
\hline 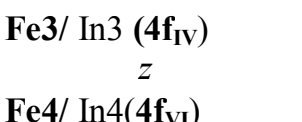 & $0.0316(5)$ & $0.0307(6)$ & $0.0311(6)$ & $0.0309(7)$ \\
\hline $\begin{array}{c}z \\
\text { Fe5/ In5 (12k) }\end{array}$ & $0.1919(5)$ & $0.1927(5)$ & $0.1935(5)$ & $0.1936(5)$ \\
\hline$x$ & $0.1837(11)$ & $0.1840(13)$ & $0.1851(12)$ & $0.1854(12)$ \\
\hline O1 (4e) & $-0.1087(1)$ & $-0.1088(2)$ & $-0.1088(2)$ & $-0.1089(2)$ \\
\hline O2 (4f) & $0.1432(5)$ & $0.1442(6)$ & $0.1438(7)$ & $0.1448(7)$ \\
\hline $\mathbf{O 3}(6 \mathrm{~h})^{z}$ & $-0.0374(6)$ & $-0.0384(7)$ & $-0.0371(7)$ & $-0.0369(8)$ \\
\hline O4 (12k) & $0.1846(34)$ & $0.1802(51)$ & $0.1723(49)$ & $0.1662(49)$ \\
\hline$x$ & $0.1604(21)$ & $0.1635(29)$ & $0.1646(27)$ & $0.1659(29)$ \\
\hline O5 (12k) & $0.0546(3)$ & $0.0546(3)$ & $0.0553(3)$ & $0.0555(3)$ \\
\hline$x$ & $0.4952(23)$ & $0.4947(27)$ & $0.4939(27)$ & $0.4938(30)$ \\
\hline$z$ & $0.1542(2)$ & $0.1538(3)$ & $0.1538(3)$ & $0.1535(3)$ \\
\hline $\mathrm{R}_{\mathrm{wp}}, \%$ & 5.34 & 6.09 & 6.07 & 6.58 \\
\hline $\mathrm{R}_{\exp }, \%$ & 3.90 & 4.01 & 3.93 & 4.38 \\
\hline $\mathrm{R}_{\mathrm{B}}, \%$ & 3.25 & 3.43 & 4.32 & 5.23 \\
\hline $\mathrm{R}_{\text {Mag, }} \%$ & 3.64 & 4.26 & 5.19 & 6.91 \\
\hline$\chi^{2}$ & 1.88 & 2.31 & 2.39 & 2.26 \\
\hline
\end{tabular}


Table 2.

Crystal structure parameters, figures of merit and atomic positions refined in the framework of centrocymmetric SG P6 ${ }_{3} \mathrm{mc}$ (No. 186) with the Rietveld method for different temperatures. Atoms are located in positions: $\operatorname{Sr}(2 \mathrm{~b})(2 / 3,1 / 3, z)$; Fe1 (2a) $(0,0, z)$; Fe2 (2a) $(0,0, z) ;(\mathrm{Fe} / \mathrm{In}) 3$ (2b) $(1 / 3,2 / 3, z) ;(\mathrm{Fe} / \mathrm{In}) 33$ (2b) $(1 / 3,2 / 3, z) ;(\mathrm{Fe} / \mathrm{In}) 4$ (2b) $(1 / 3,2 / 3, z) ;(\mathrm{Fe} / \mathrm{In}) 44(2 \mathrm{~b})$ $(1 / 3,2 / 3, z)$; (Fe/In)5 (6c) $(x, 2 x, z)$; (Fe/In)55 (6c) $(x, 2 x, z)$; O1 (2a) $(0,0, z)$; O11 (2a) $(0,0, z) ; \mathrm{O} 2(2 \mathrm{~b})(1 / 3,2 / 3, z) ; \mathrm{O} 22$ (2b) $(1 / 3,2 / 3, z)$; O3 (6c) $(x, 2 x, z)$; O4 (6c) $(x, 2 x, z)$; O44 (6c) $(x, 2 x, z)$; O5 (6c) $(x, 2 x, z)$; O55 (6c) $(x, 2 x, z)$.

\begin{tabular}{|c|c|c|c|c|}
\hline $\begin{array}{l}\text { Temperature } \\
\text { Atomic } \\
\text { positions }\end{array}$ & $50 \mathrm{~K}$ & $150 \mathrm{~K}$ & $250 \mathrm{~K}$ & $350 \mathrm{~K}$ \\
\hline$a,(\AA)$ & $5.8736(1)$ & $5.8741(2)$ & $5.8769(2)$ & $5.8807(2)$ \\
\hline$c,(\AA)$ & $22.9843(6)$ & $23.0023(7)$ & $23.0285(7)$ & $23.0581(8)$ \\
\hline$V,\left(\AA^{3}\right)$ & $686.69(3)$ & $687.35(3)$ & $688.79(4)$ & $690.58(4)$ \\
\hline $\operatorname{Sr}(2 b)$ & & & & \\
\hline Fe1 (2a) & $0.2663(14)$ & 0.2618 & 0.2587 & $0.2570(39)$ \\
\hline $\operatorname{Fe} 2(\mathbf{2 a})^{z}$ & $-0.0052(16)$ & $-0.0085(19)$ & $-0.0128(24)$ & $-0.0106(19)$ \\
\hline Fe3 (2b) & $0.2467(19)$ & $0.2459(21)$ & $0.2443(28)$ & $0.2422(49)$ \\
\hline Fe33 (2b) & $0.0261(13)$ & $0.0233(19)$ & $0.0232(19)$ & $0.0239(43)$ \\
\hline Fe4 (2b) & $0.4638(16)$ & $0.4594(26)$ & $0.4573(25)$ & $0.4618(35)$ \\
\hline Fe44(2b) & $0.1998(9)$ & $0.1949(17)$ & $0.1929(21)$ & $0.2018(26)$ \\
\hline $\operatorname{Fe} 5(6 c)^{z}$ & $0.3107(10)$ & $0.3049(23)$ & $0.3024(28)$ & $0.3087(23)$ \\
\hline$x$ & $0.1751(32)$ & $0.1759(39)$ & $0.1778(39)$ & $0.1772(45)$ \\
\hline Fe55 (6c) & $0.8880(7)$ & $0.8854(12)$ & $0.8845(13)$ & $0.8900(18)$ \\
\hline$x$ & $0.8279(31)$ & $0.8255(34)$ & $0.8209(54)$ & $0.8183(52)$ \\
\hline O1 (2a) & $0.1089(7)$ & $0.1057(11)$ & $0.1052(9)$ & $0.1104(23)$ \\
\hline O11 (2a) & $0.1520(19)$ & $0.1482(29)$ & $0.1489(29)$ & $0.1492(44)$ \\
\hline $\mathbf{O 2}(2 \mathrm{~b})^{z}$ & $0.3406(20)$ & $0.3388(28)$ & $0.3404(33)$ & $0.3491(42)$ \\
\hline O22 (2b) & 0.9375 & 0.9375 & $0.9465(69)$ & $0.9487(52)$ \\
\hline $\mathrm{O3}(\mathbf{6 c})^{z}$ & $0.5305(23)$ & $0.5273(31)$ & $0.5318(70)$ & $0.5272(69)$ \\
\hline$x$ & $0.1864(42)$ & $0.1820(68)$ & $0.1758(63)$ & $0.1749(64)$ \\
\hline $04(6 c)^{z}$ & $0.2517(10)$ & $0.2503(11)$ & $0.2511(31)$ & $0.2532(16)$ \\
\hline O44 (6c) & $\begin{array}{c}0.1806(47) \\
0.0452\end{array}$ & $\begin{array}{c}0.1810(54) \\
0.0439\end{array}$ & $\begin{array}{l}0.1797(61) \\
0.0414(12)\end{array}$ & $\begin{array}{c}0.1739(83) \\
0.0452\end{array}$ \\
\hline O5 (6c) & $\begin{array}{c}0.1420(43) \\
0.4344(9)\end{array}$ & $\begin{array}{c}0.1433(49) \\
0.4332(8)\end{array}$ & $\begin{array}{c}0.1438 \\
0.4294(13)\end{array}$ & $\begin{array}{l}0.1521(72) \\
0.4340(13)\end{array}$ \\
\hline
\end{tabular}




\begin{tabular}{|c|c|c|c|c|}
$x$ & $0.4599(39)$ & 0.4618 & $0.4637(49)$ & $0.4636(48)$ \\
$z$ & 0.1581 & $0.1529(21)$ & 0.1544 & $0.1609(33)$ \\
O55 (6c) & & & & \\
$z$ & $0.5041(57)$ & $0.5035(57)$ & $0.4981(86)$ & $0.4973(111)$ \\
& $0.3462(9)$ & $0.3432(21)$ & $0.3433(11)$ & $0.3469(32)$ \\
$\mathrm{R}_{\mathrm{wp}}, \%$ & & & \\
$\mathrm{R}_{\mathrm{exp}}, \%$ & 3.78 & 5.39 & 5.30 & 5.54 \\
$\mathrm{R}_{\mathrm{B}}, \%$ & 3.99 & 3.90 & 4.35 \\
$\mathrm{R}_{\mathrm{Mag}}, \%$ & 1.64 & 1.80 & 2.49 & 2.69 \\
$\chi^{2}$ & 1.90 & 1.96 & 3.05 & 3.99 \\
\hline
\end{tabular}

